\title{
MILD INDUCED HYPOTHERMIA AND AN URGENT INVASIVE CORONARY STRATEGY - A PROMISING PROTOCOL FOR COMATOSE SURVIVORS OF SUDDEN CARDIAC ARREST
}

Marko Noc, MD, PhD, FESC

Center for Intensive Internal Medicine, University Medical Center

\section{INTRODUCTION}

Sudden cardiac arrest remains the leading cause of death in developed countries, with an annual incidence ranging from 36 to 81 events per 100.000 inhabitants. Following the initial cardiopulmonary resuscitation, spontaneous circulation can be restored in 40 to $60 \%$ of the patients. Because of the usual delays in the "chain of survival", more than $70 \%$ of resuscitated patients typically remain comatose upon hospital admission. This is due to postresuscitation brain injury, which may vary in severity from mild disability to a permanent vegetative state. Because no effective treatment was available in the past, the great majority of comatose survivors of cardiac arrest ultimately died in the hospital or in nursing homes in a permanent vegetative state.

The era of mild induced hypothermia, which began in 2002 following the landmark publication of two independent randomized trials $(1,2)$, undoubtedly revolutionised the field of postresuscitation treatment. Indeed, with a number required to treat between 7 and 8 , hypothermia is a unique intervention in modern cardiovascular medicine. After effective treatment for postresuscitation brain injury became available and comatose patients "started to wake up" during subsequent days of treatment, more efforts have been made to define and treat the cause of cardiac arrest. Because an acute coronary event leading to critical narrowing or complete coronary obstruction is the main trigger of sudden cardiac arrest, urgent coronary angiography followed by percutaneous coronary intervention (PCI) has been increasingly performed upon hospital admission $(3,4)$. We have learned that urgent PCI is feasible, safe, and successful and may improve the survival of patients with resuscitated cardiac arrest. Several hospitals have therefore designed dedicated postresuscitation protocols for comatose survivors of cardiac arrest that incorporate mild induced hypothermia, an urgent invasive coronary strategy and intensive care support related to haemodynamics, respiration, and electrolyte and acid-base balance (5).
Evolution of postresucitation management of comatose survivors of cardiac arrest at the University Medical Center in Ljubljana

We used mild induced hypothermia for the first time in September 2003, and since then, it has quickly become the standard of care in comatose survivors of cardiac arrest. Our usual hypothermia protocol is simple and cheap and can be immediately implemented in every hospital (Figure 1). In short, comatose survivors of cardiac arrest are obviously intubated and mechanically ventilated. After achieving the appropriate sedation and muscle relaxation to prevent shivering, hypothermia is induced by the rapid infusion ( $30 \mathrm{ml} / \mathrm{kg}$ in 30 minutes) of cold saline at $4 \mathrm{C} \mathrm{(6).}$ Ice packs are simultaneously used to augment cooling. Using this method, we are able to reach a target central temperature between 32 and $34 \mathrm{C}$ in 3 to 4 hours. This target temperature, measured by urinary catheter, is then maintained for 24 hours and followed by spontaneous rewarming, which should not exceed $0.5 \mathrm{C}$ per hour. During and following rewarming, it is very important to prevent temperature rise, shivering and hypovolemia. We initially started the hypothermia protocol only after hospital admission. However, after gaining more experience, we advised our prehospital emergency units and referring hospitals to start hypothermia immediately after re-establishing spontaneous circulation and continue during the transport to our hospital.

Because we are a primary PCI centre for ST-elevation myocardial infarction (STEMI) with 24-hour service since 2000 , we gradually adopted a strategy of urgent coronary angiography and PCI in comatose survivors of cardiac arrest. History of coronary artery disease, chest discomfort before the onset of cardiac arrest, signs of STEMI or other ischemic changes in the postresuscitation electrocardiogram (ECG) argue for an acute coronary cause of arrest and urgent coronary angiography to immediately define the anatomical lesion(s). Indeed, the lesion may be found not only in a high percentage of 
Figure 1. Protocol for mild induced hypothermia in comatose survivors of cardiac arrest at the University Medical Centreer in Ljubljana (Slovenia)
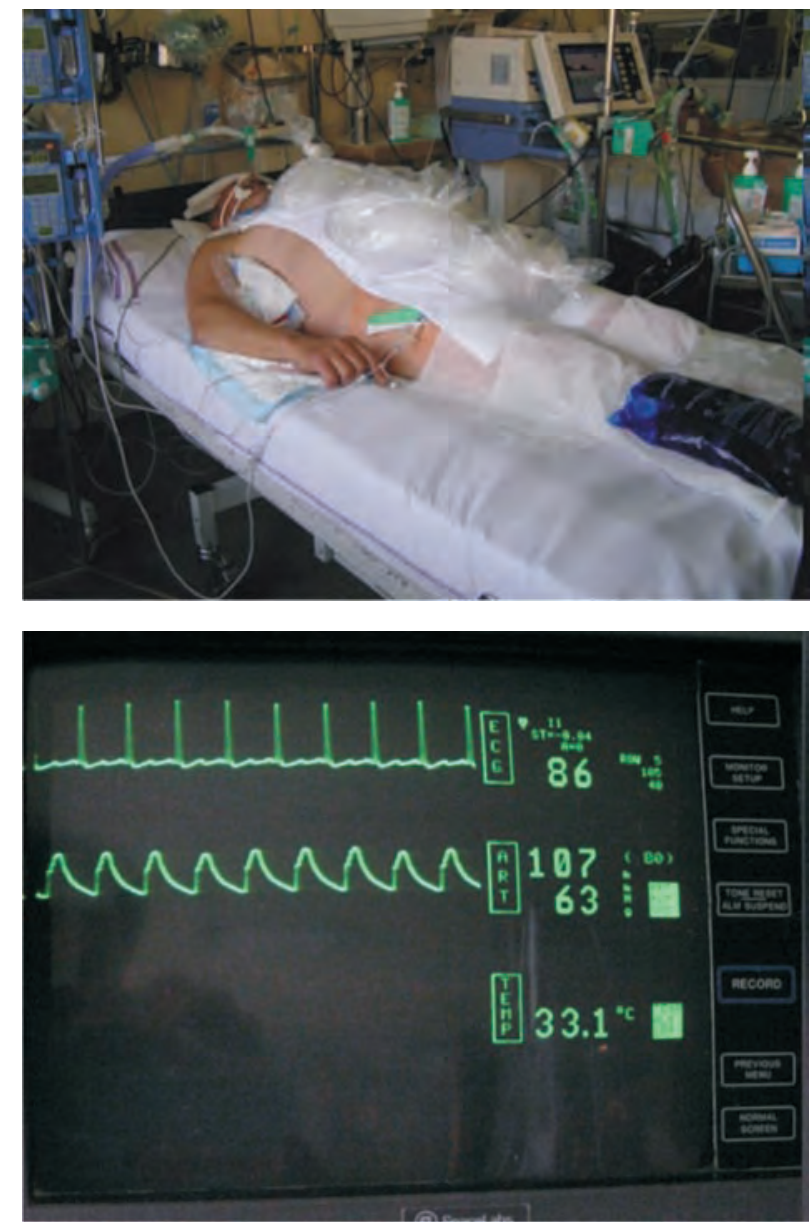

- Intubation /mechanical ventilation

- Sedation (midazolam 0.1-0.3mg/kg bolus+infusion)

- Muscle relaxation

(0.1 mg/kg norcuronium + repeated when shivering)

- Rapid infusion of $0.9 \% \mathrm{NaCl}$ at $4 \mathrm{C}$

(30 ml/kg in 30 minutes)

- Ice packs (head, neck, axilla, abdomen)

- Maintain 32-34 C for 24 hours

(Urinary bladder temperature)

Start hypotermia after reestablishment of spontaneous circulation already on the field

\section{Urgent transport to dedicated} interventional cardiology center with skilled cardiac intensive care unit patients with STEMI but also in up to $30 \%$ of patients without STEMI in a postresuscitation ECG $(3,4)$. We also demonstrated that combining urgent invasive coronary strategy with hypothermia is feasible and safe (6). Hypothermia does not compromise the angiographic result of $\mathrm{PCI}$, and there is no excess in arrhythmias and haemodynamic instability requiring more aggressive support with inotropes, vasopressors or an intra-aortic balloon pump $(6,7)$. Moreover, when the proportion of comatose survivors of out-of-hospital cardiac arrest undergoing hypothermia and urgent invasive coronary strategy increased from $0 \%$ between $1995-97$ to $90 \%$ and $70 \%$ between 2006 and 2008, respectively, the survival to hospital discharge concomitantly increased from $24 \%$ to $62 \%$. Importantly, survival with good neurological recovery concomitantly increased from $15 \%$ to $40 \%$. These impressive but not yet "peer review" published results were independently confirmed by other investigators using the same strategy of aggressive postresuscitation management $(5,8)$. We therefore designed a special "fast track" for comatose survivors of cardiac arrest and complemented the already existing "STEMI-primary PCI" network to offer the benefits of this treatment to patients from remote areas (Figure 2).

\section{CONCLUSION}

Mild induced hypothermia and an urgent invasive coronary strategy on suspicion of a coronary cause of cardiac arrest should be part of a comprehensive postresuscitation treatment protocol for comatose survivors of cardiac arrest. Such an "organ-oriented" and aggressive treatment strategy may significantly improve the previously dismal survival rates in these patients. It is likely that the best results may be achieved if the treatment of comatose survivors of cardiac arrest is centralised to dedicated interventional cardiology centres that already have a "STEMI-primary PCI" network with a high volume of acute $\mathrm{PCI}$ procedures and a competent cardiac intensive care unit.

Urgent coronary
angiography-
PCI if coronary
cause is likely
while hypo-
thermia is
ongoing

Complete 24-hour hypotermia and provide intensive care support

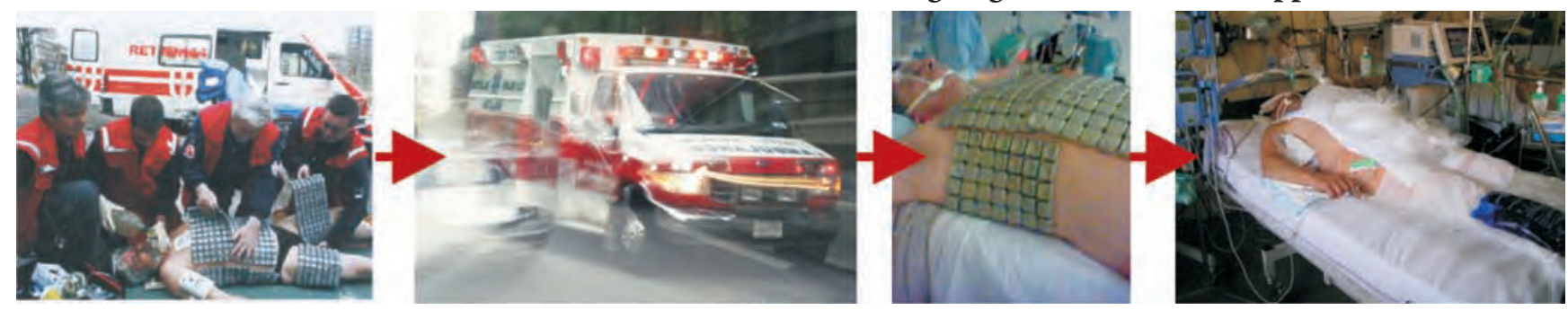

Figure 2. "Fast track" for comatose survivors of out-of-hospital cardiac arrest. 


\section{REFERENCES}

1. The Hypothermia After Cardiac Arrest (HACA) study group. Mild therapeutic hypothermia to improve the neurological outcome after cardiac arrest. N Engl J Med 2002;346:549-56.

2. Bernard SA, Gray TW, Buist MD, Jones BM, Silvester W, Gutteridge G, Smith K. Treatment of comatose survivors of out-of-hospital cardiac arrest with induced hypothermia. N Engl J Med 2002;346:557-63.

3. Spaulding CM, Joly LM, Rosenberg A, Monchini M, Weber SN, Dhainaut JA, Carli P. Immediate coronary angiography in survivors of out-of hospital cardiac arrest. N Engl J Med 1997;336:1629-33.

4. Dumas F, Cariou A, Manzo-Silberman S, Grimaldi D, Vivien B, Rosencher J, Empana JP, Carli P, Mira JP, Jouven $\mathrm{X}$, Spaulding $\mathrm{C}$. Immediate percutaneous coronary intervention is associated with better survival after out- of-hospital cardiac arrest. Insights from the PROCAT registry. Circ Cardiovasc Interv 2010;3:200-7.

5. Sunde K, Pytte M, Jacobsen D, Mangschau A, Jensen LP, Smedsrund C, Draegni T, Steen PA. Implementation of a standardized treatment protocol for post resuscitation care after out of-hospital cardiac arrest. Resuscitation 2007;73:29-39.

6. Knafelj R, Radsel P, Ploj T, Noc M. Primary percutaneous coronary intervention and mild induced hypothermia in Comatose survivors of ventricular fibrillation with ST-elevation acute myocardial infarction. Resuscitation 2007;74:227-234.

7. Wolfrum S, Pierau C, Radke PW, Schunkert H, Kurowski V. Mild therapeutic hypothermia in patients after out-ofhospital cardiac arrest due to acute ST-segment elevation myocardial infarction undergoing immediate percutaneous coronary intervention. Crit Care Med 2008;36:1780-86.

8. Stub D, Hengel C, Chan W, Jackson D, Sanders K, Dart AM, Hilton A, Pellegrino V, Shaw JA, Duffy SJ, Bernard S, Kaye DM. Am J Cardiol 2011;107:522-7.

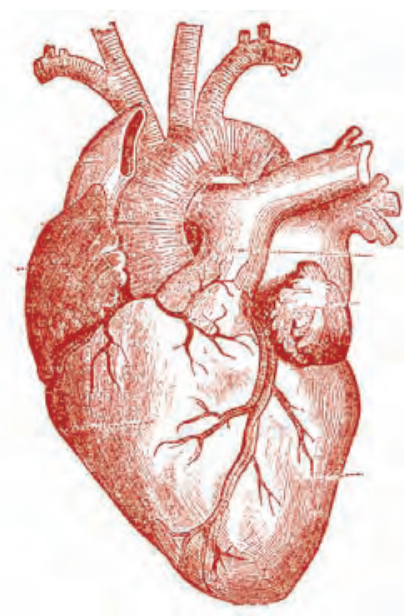

